\title{
Job Burnout Among Family Physicians in Rural Areas of Isfahan Province
}

\author{
Neda Moein ${ }^{1}$, Gholamhossein Ahmadzadeh ${ }^{2 *}$, Alireza Safaeeyan ${ }^{1}$ \\ 'Department of Community Medicine and Family Medicine, Isfahan University of Medical Sciences, Isfahan, Iran \\ ${ }^{2}$ Department of Psychiatry, Isfahan University of Medical Sciences, Isfahan, Iran
}

*Corresponding Author: Gholamhossein Ahmadzadeh, M.D., Associated Professor, Department of Psychiatry, Isfahan University of Medical Sciences, Isfahan, Iran. Tel: +98-313-2222135, Email: ahmadzadeh@med.mui.ac.ir

Received March 17, 2018; Accepted May 29, 2018; Online Published June 17, 2018

\begin{abstract}
Background: In providing optimum medical and health services, great pressure is put on the physical and mental health of family physicians. Job burnout is damaging to the health of family physicians and medical treatment personnel. It leads to reduced job productivity, increased absenteeism, increased healthcare costs, elevated turnover rates, a reduced level of service provided to patients, and ultimately, patient dissatisfaction.

Objective: The current research investigated job burnout among family physicians in rural areas of Isfahan province during the years 2017-2018.

Methods: This cross-sectional study was carried out in Isfahan province during the years 2017-2018. The research population included all family physicians working in Isfahan province, and 155 of whom met the inclusion criteria and participated in this research. Questionnaires were used as the data collection tool. Data was analyzed using SPSS software, and the analytical variables were analyzed using the independent $t$ test and Pearson correlation coefficient.

Results: A total of 45 men (29\%) and 110 women (71\%) comprised the research population. Participants' mean age and mean duration of work experience were $35.3 \pm 8.1$ and 7.5 years, respectively. The scores for overall job burnout, emotional exhaustion, depersonalization, and lack of personal accomplishment dimensions were low among the family physicians in Isfahan. The results showed no significant difference between male and female, single and married participants in any of the dimensions. The results also revealed a direct relationship between years of work experience and the overall job burnout and lack of personal accomplishment scores.

Conclusion: According to the findings of the present research and considering the stressful nature of a family physician's job, healthcare authorities need to pay special attention to job burnout and implement measures to prevent it or at least reduce its subsequent adverse effects.
\end{abstract}

Keywords: Job Burnout, Family Physicians, Health, Mental Health

\section{Background}

A major portion of each individual's daily life is spent doing a job. Various factors, including environmental (noise, overcrowding, inadequate lighting and sound), human factors (conflict with other individuals), and organizational (inappropriate workload and policymaking) aspects have always existed as stressors in the workplace. The incapacity of a person to effectively cope with these mental stressors leads to the development of many physical, mental, and behavioral complications. Long-term exposure to these mental stressors leads to reduced job satisfaction and job burnout syndrome. The symptoms of this syndrome manifest when one is incapable of meeting the demands of the workplace. They impose negative effects on one's social, physical, and psychological life. Three dimensions of job burnout are: (1) Emotional exhaustion (chronic exhaustion, sleep disorders, various physical symptoms) that manifests itself in the form of reduced energy and feeling mentally drained; (2) Depersonalization (negative, emotionless, and excessive disregard of colleagues and clients; feelings of guilt and isolation; reduced job productivity and daily activity), meaning that a person is mentally detached from his/her job; and (3) Lack of personal accomplishment (reduced competence and job accomplishment, job dissatisfaction, feelings of failure and hopelessness, losing the power to recognize and understand, permanent feelings of extortion and exploitation, reduced job performance). ${ }^{1-3}$ Previous research has shown that job stresses lead to job burnout. There is evidence confirming that medicine is a stressful profession. ${ }^{4,5}$ Due to frequent exposure to stresses such as observing patient suffering and death, conflicting with others, having low social support, experiencing excessive job stress, being exposed to a high number of patients per day, making emergency decisions based on

Copyright $(\subset) 2018$ The Author(s). This is an open-access article distributed under the terms of the Creative Commons Attribution License (http:// creativecommons.org/licenses/by/4.0), which permits unrestricted use, distribution, and reproduction in any medium, provided the original work is properly cited. 
insufficient information and assuming accountability for the outcomes arising from this decision, experiencing violence and threats in the workplace, working night shifts, and dealing with professional responsibilities and the issues related to the administrative system, people in the medical field are more prone to job burnout syndrome than those in other professions. ${ }^{6-9}$ Job burnout imposes many complications on the individual and one's family, social, and organizational life, the most important of which are absenteeism and turnover, frequent delays, various psychological complaints, conflicts, job changes, poor quality of care, unwillingness to take care of a patient, and conflicts with colleagues. ${ }^{10,11}$ Job burnout has a close relationship with absenteeism and turnover, especially among nurses. It ultimately leads to reduced attention and care for patients. ${ }^{12}$ Navidian et al reported a moderate burnout rate among the target population of the study and found a significant correlation between job burnout and general health. ${ }^{13}$ Rahmani et al, ${ }^{14}$ Dick, ${ }^{15}$ and Oohler et al ${ }^{16}$ also found a significant correlation between job burnout and general health in their studies. Anisi et $\mathrm{al}^{17}$ and Van Horn et $\mathrm{al}^{18}$ also observed a significant correlation between demographic characteristics such as age and years of work experience and job burnout. Dick ${ }^{15}$ referred to job burnout as a feeling of being exploited and excessive exhaustion which does not go away by resting or taking a leave of absence. Dick ${ }^{15}$ further concluded that job burnout is a state in which the individual assumes s/he is less valuable than others and feels that his/her job and performance are neglected by others. McCray et al performed a systematic review about job burnout among physicians from 1966 to 2007, in the United States. Out of 190 existing articles, 129 articles were reviewed. The results indicated that the rate of job burnout fluctuated between $43 \%-45 \%$ among medical students, 22\%-60\% among specialists and general practitioners, and 56\%-80\% among family physicians. ${ }^{19}$

\section{Objective}

The present study investigated the rate of job burnout among family physicians working in rural health centers of Isfahan province during 2017-2018.

\section{Methods}

The present research is a cross-sectional study. The research population comprised all family physicians working in rural areas of Isfahan province that were distributed in 23 health centers (197 health home) of the province. The inclusion criteria used were working in a rural area for a minimum of 6 months on a family physician's contract, not having already completed the questionnaire, and giving personal consent to participate in the study. Two questionnaires were used in the study. Furthermore, the mental stress levels of all subjects were evaluated using the Holmes-Rahe Stress Inventory. Physicians who had suffered severely stressful events (death of a spouse or other family member, living apart from spouse, divorce, injury, or disease) within the preceding 12 months were not enrolled in the study. Individuals who did not complete the questionnaire or withdrew from the family physician plan were also excluded from the study. A total of 155 participants completed the questionnaires which were used for statistical analysis. Considering the distance between the provincial health centers, to save costs, and to expedite the study, the questionnaires were delivered to the Province's Health Services Development Unit, from where they were sent to the main development unit of the health centers and on to the development unit of each center where the authorities distributed them among those family physicians who met the inclusion criteria. Ten days later, the questionnaires were collected. The first questionnaire collected demographic information, including gender, age, marital status, type of employment, length of employment, length of stay in the current workplace, population covered, number of daily shifts, overnight stay, distance between the work place and residence, salary, attendance in the Master of Public Health (MPH) course, and employment at other work centers (private office or emergency department). The second questionnaire included the Maslach Burnout Inventory (MBI), the most common tool for assessing job burnout. This inventory consists of 22 statements and measures all 3 dimensions of job burnout, with 9, 5, and 8 statements for the emotional exhaustion, depersonalization, and lack of personal accomplishment dimensions, respectively. The frequency of these emotions is measured by scores ranging from 0 (never) to 6 (every day). The scores obtained in each of these three dimensions are categorized as low, medium, or high. Scores greater or equal to $27,17-26$ and lower, or equal to 16 indicate high, moderate, and low levels of emotional exhaustion, respectively. Scores greater or equal to 13, 7-12 and lower, or equal to 6 indicate high, moderate, and low levels of depersonalization, respectively. Finally, scores greater and equal to $31,32-38$ and lower, or equal to 39 demonstrate high, moderate and low levels of a lack of personal accomplishment, respectively. ${ }^{20}$ Generally, high emotional exhaustion and depersonalization scores, and a low lack of personal accomplishment score reflect job burnout. The validity and reliability of the MBI were first approved in Iran by Filian who used the test-retest method and reported a reliability coefficient of $0.78 .^{21}$ Questionnaires were studied and analyzed, and the data was later entered into the software SPSS version 20. Descriptive statistics were then used to examine and analyze descriptive variables, and analytical variables were analyzed using the independent $t$ test and Pearson correlation coefficient. A $P$ value $<0.05$ was considered as statistically significant during the data analysis phase.

\section{Results}

The mean age of participants was $35.3 \pm 8.5$ years ( $M i n=25$ years and $M a x=29$ years), and the average number of years of work experience was $7.55 \pm 7$ with a minimum of 6 months and maximum of 29 years. The values of the other quantitative variables are listed in Table 1. 
Table 1. The Values of Different Quantitative Variables

\begin{tabular}{|c|c|c|c|c|c|}
\hline Variable & Mean & SD & Median & Minimum & Maximum \\
\hline Age $(y)$ & 35.3 & 8.5 & 34 & 25 & 53 \\
\hline Work experience $(y)$ & 7.55 & 7 & 5.55 & 0.5 & 29 \\
\hline Length of stay at current workplace (y) & 3.26 & 4.3 & 1.3 & 0.08 & 24 \\
\hline The number of population covered & 4508 & 2233 & 4300 & 699 & 13000 \\
\hline The number of daily shifts & 3 & 1.6 & 3 & 1 & 9 \\
\hline Distance between workplace and residence $(\mathrm{km})$ & 68.3 & 86.9 & 30 & 0.2 & 450 \\
\hline
\end{tabular}

Table 2 shows that $71 \%$ of the subjects are women and $69.7 \%$ of the subjects are married. The percentages of subjects who had an overnight stay, work in other centers, and had a history of attending MPH programs held for family physicians is $18.7 \%, 7.1 \%$, and $18.1 \%$, respectively.

As shown in Table 3, 47.1\% of physicians had low emotional exhaustion scores, and the mean job burnout score in this area was 19.1 out of 54. The majority of participants $(81.3 \%)$ were categorized as having low job burnout in the depersonalization dimension. It should be noted that the mean job burnout score of the subjects in this dimension was 3.9 out of 30 . A total of $47.7 \%$ of the participants obtained a low score in the lack of personal accomplishment dimension with the mean score being 12.4 out of 48 .

The results of the correlation coefficient test showed the direct correlation of a lack of personal accomplishment with work experience $(\mathrm{r}=0.175, P=0.03)$ and length of stay in the current workplace $(\mathrm{r}=0.168, P=0.046)$ (Table 4$)$. In

Table 2. Demographic Characteristics of the Physicians Studied

\begin{tabular}{lc}
\hline Variables & Number $(\%)$ \\
\hline Gender & $110(71)$ \\
Female & $45(29)$ \\
Male & \\
Marital status & $47(30.3)$ \\
Single & $108(69.7)$ \\
Married & $29(18.7)$ \\
Overnight stay & $11(7.1)$ \\
Concurrent employment at other centers & $28(18.1)$ \\
\hline Family physician attended MPH courses &
\end{tabular}

other words, the more work experience a physician has and the longer the physician's stay is at the current location, the higher the lack of personal accomplishment score will be. The results of the correlation coefficient test showed no significant correlation between work experience and emotional exhaustion or depersonalization score, and no significant correlation between the other quantitative variables and the job burnout dimensions $(P>0.05)$.

The results showed no significant correlation between the gender of the physicians and their job burnout score; that is to say, there was no significant difference between men and women in terms of the job burnout rate in emotional exhaustion $(P=0.77)$, depersonalization $(P=0.43)$, or lack of personal accomplishment $(P=0.69)$ dimensions. The results also showed no significant relationship between the marital status of the physicians and their job burnout score (Table 5); that is to say, there was no significant difference between married and single individuals in terms of job burnout score in the emotional exhaustion $(P=0.14)$, depersonalization $(P=0.34)$, and lack of personal accomplishment $(P=0.41)$ dimensions. There was no significant difference between those who had an overnight stay and those who did not in terms of the mean emotional exhaustion $(P=0.20)$ and depersonalization $(P=0.87)$ scores; however, the lack of personal accomplishment scores were significantly higher among those who had an overnight stay $(P=0.009)$. There was no significant difference between the overall job burnout score in the emotional exhaustion $(P=0.62)$ and lack of personal accomplishment $(P=0.61)$ dimensions among people who work and those who do not work in other centers, but the depersonalization score was significantly higher among those who work in other centers $(P=0.01)$. There

Table 3. Frequency distribution of job burnout in different dimensions

\begin{tabular}{llll}
\hline Variable & Level & Number (\%) & Maximum Score \\
\hline \multirow{2}{*}{ Emotional exhaustion } & Low (less than or equal to 16) & $73(47.1)$ & 54 \\
& Medium (17 to 26) & $47(30.3)$ & $35(22.6)$ \\
& High (27 and above) & $126(81.3)$ & 3.1 \\
Lew (less than or equal to 6) & $24(15.5)$ & $5(3.2)$ & 30 \\
Depersonalization & Average (7 to 12) & $74(47.7)$ & 48 \\
Lack of personal & High (13 and above) & $37(23.9)$ & 12.4 \\
accomplishment & Medium (32 to 38) & $44(28.4)$ & 4.9 \\
\hline
\end{tabular}


Table 4. Pearson and Spearman Correlation Coefficients Between Different Quantitative Variables and the Overall Job Burnout Score With its Dimensions

\begin{tabular}{|c|c|c|c|c|c|c|}
\hline \multirow{2}{*}{ Variable } & \multicolumn{2}{|c|}{ Emotional Exhaustion } & \multicolumn{2}{|c|}{ Depersonalization } & \multicolumn{2}{|c|}{ Lack of Personal Accomplishment } \\
\hline & $\boldsymbol{P}$ & $R$ & $\boldsymbol{P}$ & $\boldsymbol{R}$ & $\boldsymbol{P}$ & $\boldsymbol{R}$ \\
\hline Age $(y)$ & 0.049 & 0.57 & 0.016 & 0.85 & 0.119 & 0.16 \\
\hline Work experience $(y)$ & 0.099 & 0.24 & 0.138 & 0.09 & 0.175 & 0.03 \\
\hline Length of stay at current workplace (y) & 0.016 & 0.85 & -0.064 & 0.45 & 0.168 & 0.046 \\
\hline The number of population covered & -0.048 & 0.57 & 0.006 & 0.94 & -0.028 & 0.74 \\
\hline The number of ambulation & -0.111 & 0.30 & -0.004 & 0.97 & -0.086 & 0.42 \\
\hline Distance between the workplace and place of residence $(\mathrm{km})$ & -0.075 & 0.40 & 0.104 & 0.24 & -0.134 & 0.13 \\
\hline
\end{tabular}

Table 5. Average Overall Job Burnout Score and its Dimensions by Demographic Variables

\begin{tabular}{|c|c|c|c|c|c|c|c|}
\hline \multirow{2}{*}{ Variable } & & \multicolumn{2}{|c|}{ Emotional Exhaustion } & \multicolumn{2}{|c|}{ Depersonalization } & \multicolumn{2}{|c|}{ Lack of Personal Accomplishment } \\
\hline & & Mean & SD & Mean & SD & Mean & SD \\
\hline \multirow{2}{*}{ Sex } & Female & 19.3 & 10.4 & 3.7 & 1.5 & 12.6 & 7.6 \\
\hline & Male & 18.8 & 10.9 & 4.2 & 2.2 & 12.03 & 8.9 \\
\hline \multicolumn{2}{|l|}{$P$ (independent sample $t$ test) } & \multicolumn{2}{|l|}{0.770} & \multicolumn{2}{|l|}{0.430} & \multicolumn{2}{|l|}{0.690} \\
\hline \multirow{2}{*}{ Marital Status } & Single & 17.2 & 8.9 & 4.3 & 1.9 & 11.6 & 8.2 \\
\hline & Married & 19.9 & 11.1 & 3.7 & 1.6 & 12.7 & 7.9 \\
\hline \multicolumn{2}{|l|}{$P$ (independent sample $t$ test) } & \multicolumn{2}{|l|}{0.140} & \multicolumn{2}{|l|}{0.340} & \multicolumn{2}{|l|}{0.410} \\
\hline \multirow{2}{*}{ Place } & Yes & 16.9 & 9.2 & 4.0 & 1.7 & 9 & 6.3 \\
\hline & No & 19.6 & 10.7 & 3.8 & 1.8 & 13.2 & 8.1 \\
\hline \multicolumn{2}{|l|}{$P$ (independent sample $t$ test) } & \multicolumn{2}{|l|}{0.201} & \multicolumn{2}{|l|}{0.780} & \multicolumn{2}{|l|}{0.009} \\
\hline \multirow{2}{*}{ Employment at other centers } & Yes & 20.6 & 12.6 & 6.5 & 3.3 & 11.2 & 6.5 \\
\hline & No & 19.01 & 10.4 & 3.7 & 1.4 & 12.5 & 8.1 \\
\hline \multicolumn{2}{|l|}{$P$ (independent sample $t$ test) } & \multicolumn{2}{|l|}{0.620} & \multicolumn{2}{|l|}{0.010} & \multicolumn{2}{|l|}{0.610} \\
\hline \multirow{2}{*}{ Attend MPH course family physician } & Yes & 20.5 & 13.3 & 3.88 & 1.2 & 15.0 & 8.7 \\
\hline & No & 18.8 & 9.8 & 3.86 & 1.9 & 11.8 & 7.7 \\
\hline$P$ (independent sample $t$ test) & & \multicolumn{2}{|l|}{0.460} & \multicolumn{2}{|l|}{0.970} & \multicolumn{2}{|l|}{0.057} \\
\hline
\end{tabular}

was no significant difference between those who attend and those who did not attend the MPH course in terms of the overall job burnout score in the emotional exhaustion $(P=0.46)$, depersonalization $(P=0.97)$, or lack of personal accomplishment $(P=0.057)$ dimensions.

\section{Discussion}

The present study showed that the job burnout rate is low in the emotional exhaustion, depersonalization, and lack of personal accomplishment dimensions among family physicians working in rural health centers of Isfahan province. One study among family physicians in Khorasan Razavi province (2013), Mahdizadeh et al showed that the job burnout rate was at a moderate level in the lack of personal accomplishment and depersonalization dimensions and high in the emotional exhaustion dimension, ${ }^{22}$ which is not consistent with the present study. This study showed that a high percentage of family physicians were not satisfied with the mismatch between income and working time, workload, and overnight stay conditions. Abdulghafour showed a significant correlation between the different dimensions of job burnout and level of income and the distance between residence and the workplace. ${ }^{23}$ Similarly, Masoudi et al showed that the most important job burnout-inducing factors include the mismatch between salary and the work done, the low probability of progress, being accountable to a large number of clients during the day, and the excessive expectations of clients and their families. ${ }^{24}$ Mahmoodi et al also showed a significant correlation of emotional exhaustion and depersonalization with the physical conditions of the work environment as well as specialized facilities of the work environment. ${ }^{25}$ The legal status of family physicians as well as the overnight stay facilities and conditions have been improved considerably since 2014 (i.e. about 9 years after the start of the family doctor plan). These factors can explain the discrepancy between the 2 studies and also confirm the fact that the job burnout rate can be effectively reduced by establishing a balance between salary and workload and by improving the physical environment of the workplace. Koys showed in his study that nearly $50 \%$ of nurses experience high levels of job burnout. ${ }^{26}$ Talaei et al also showed that the job burnout rate was high among employees of Mashhad's medical treatment centers. ${ }^{27}$ These findings are not consistent with the current study. The discrepancy can be due to the different work environment existing in health centers and medical treatment centers. Although treatment-related activities are also carried out in rural health centers, health personnel suffer from much less stress caused by the death of a patient and 
the fatigue resulting from work shifts compared to the medical treatment personnel. The results of the present research showed a direct relationship between the work experience of family physicians and their lack of personal accomplishment. This could be due to fatigue and the lack of scientific achievement as well as the reduced physical and psychological abilities of physicians due to increasing age. The emotional exhaustion rates of $47.1 \%$ and $53 \%$ of family physicians were reported to be at low and moderateto-high levels, respectively. Multiple confrontations with stressful scenes, patients' deaths, and the responsibility of caring for patients with special conditions are among the factors that gradually lead to emotional exhaustion and reduce the ability to support patients and provide optimal patient care. Therefore, empowering family physicians with the skills to cope with stressors not only pave the way for reducing or even eliminating these factors, but it can also play a role in creating a quiet work environment, increasing capacity and efficiency rates, ${ }^{28}$ and reducing the physicians' levels of emotional exhaustion. The depersonalization rates were reported to be low, moderate, and high among $81.3 \%, 15.5 \%$, and $3.2 \%$ of family physicians. This job burnout dimension is characterized by a non-emotional and yet aggressive response to clients and colleagues. A person with job burnout syndrome holds negative feelings and attitudes, blame others, and perceive people as nonhuman objects; for example, physicians with job burnout view their patients as objects and recognize them with type of disease, room number, etc. They do not feel the pain of the patients. In a study on ICU personnel, Frade et al reported the levels of emotional exhaustion and lack of personal accomplishment to be at the moderate level and depersonalization at high level. He also measured the severity of job burnout as $14 \%{ }^{29}$ Santana et al found that there is a high level of emotional exhaustion and depersonalization in all nurses' groups. ${ }^{30}$ Furthermore, Mahdizadeh et al reported that family physicians suffer from moderate depersonalization, ${ }^{22}$ which is not consistent with the present study. The indifference to people and the lack of attaching importance to the client's fate are among the options included in the questionnaire on the depersonalization dimension. In these areas, the length of stay of family physicians and working for a specific population seems to be very effective. The continuity of the service provided is much less common among the medical staff. Mahdizadeh et al also reported that the average work experience of physicians was 3 years, ${ }^{22}$ and the average length of stay was 7.5 years in this study. The longer the length of stay is, the greater the familiarity and intimacy between the physician and the people will be, and the client's fate becomes more important for the service provider. These cases can justify the discrepancy among these studies. A total of $47.7 \%$ and $51.3 \%$ of family physicians were low and moderate-high in terms of lack of personal accomplishment, respectively. Lack of personal accomplishment was associated with a reduction in the sense of competence and success in one's profession,

\section{Research Highlights}

\section{What Is Already Known?}

Job burnout reduces productivity, increases in absenteeism, increased health costs and staff turnover, and reduces patient service and dissatisfaction. Also job burnout is high among family physicians.

\section{What This Study Adds?}

This study showed that improving the economic and welfare status would reduce the amount of burnout among family physicians.

dissatisfaction with the work, a sense of failure and inability, and a sense of being threatened and exploited. The results of the current study revealed that more work experience and the length of the stay in the current workplace leads to elevated levels of lack of personal accomplishment. Considering that the length of stay is both necessary for family physicians and for the population covered, paying more attention to the provision of facilities for the work environment and to giving more autonomy to family physicians over the population covered will help reduce the level of lack of personal accomplishment.

One of the limitations of the present study was the selfreporting completion of the questionnaires; participants may exaggerate or understate their feelings or the conditions. Nevertheless, these findings and comparing them with those of similar studies carried out across the country can help identify the stressors and work environment indices affecting job burnout so as to initiate positive organizational changes. It is recommended that further research be carried out to determine other causes of burnout (such as job satisfaction), how to control burnout, and effective intervention strategies to reduce its subsequent adverse effects in the future.

\section{Conclusion}

The findings of the present research showed that job burnout among family physicians has decreased compared to an identical study carried out three years ago. This is probably due to recent changes in income levels and recently improved welfare facilities in recent years. Nevertheless, the burnout rate is still moderate to high in some individuals. In order to advance organizational goals and improve the quantity and quality of services, the burnout rate must be further reduced among family physicians by training them on how to cope with work stress, providing them with optimal working conditions, providing health personnel with social and organizational support and the opportunity to address daily affairs and leisure activities, and providing the ground for promotions and the achievement of capabilities through training programs. Considering the important nature of family physicians in promoting public health, managers as well as health authorities need to pay special attention to job burnout and 
its levels among family physicians. Furthermore, they need to present and implement strategies for the prevention or, at least, reduction of subsequent adverse effects.

\section{Authors' Contributions}

All authors contributed equally to the study.

\section{Conflict of Interest Disclosures}

The authors declare that they have no conflicts of interest.

\section{Ethical Approval}

This study was approved by the Isfahan University of Medical Sciences ethics committee with code "Ir.mui. rec.1394.3.020".

\section{Acknowledgments}

The authors would like to express their appreciation to the respected physicians participating in this study and the authorities responsible for the Development and Coordination Units of the healthcare centers in the cities of Isfahan who helped in carrying out this research project (No. 394020).

\section{References}

1. Ozyurt A, Hayran O, Sur H. Predictors of burnout and job satisfaction among Turkish physicians. QJM. 2006;99(3):161169. doi:10.1093/qjmed/hcl019.

2. Maslach C. Burnout: A social psychological analysis. Human Behavior. 1976;5:16-22.

3. Maslach C, Goldberg J. Prevention of burnout: New perspectives. Appl Prev Psychol. 1998;7(1):63-74. doi:10.1016/ S0962-1849(98)80022-X.

4. Baraz Pordanjani S, karimipor F, Ebrahimi Dastgerdi M. Evaluation of the relationship between self - esteem and burnout in nurses of hospitals of Ahvaz Jundishapur University of Medical Sciences. Iran J Nurs Res. 2012;7(27):44-51.

5. Rajaei M, Haghighi H, Dadipoor S, et al. Relationship between occupational stress and job satisfaction among Iranian obstetricians. Hormozgan Med J. 2013;17(3):249-255.

6. Moein M, Adib Hajbaghery M. Comparison of Occupational Stress among Female Nurses and Female Members of the Medical Group in Chosen Training Hospitals in Isfahan. J Nurs Educ. 2015;3(2):1-10.

7. Roberts R, Golding J, Towell T, Weinreb I. The effects of economic circumstances on British students' mental and physical health. J Am Coll Health. 1999;48(3):103-109. doi:10.1080/07448489909595681.

8. Romani M, Ashkar K. Burnout among physicians. Libyan J Med. 2014;9:23556.doi:10.3402/ljm.v9.23556.

9. Wu S, Zhu W, Wang Z, Wang M, Lan Y. Relationship between burnout and occupational stress among nurses in China. J Adv Nurs. 2007;59(3):233-239. doi:10.1111/j.13652648.2007.04301.x.

10. Gorrell BJ, Langenbach M. Curriculum issues related to nurse turnover. J Nurs Educ. 1994;33(3):101-106. doi:10.3928/01484834-19940301-04.

11. Hakim Shooshtari M, Khajeholdin N, Arabgol F, Ellahi F. The relationship between nurse burnout and their infant behavior problems. Advances in Cognitive Sciences. 2004;6(1-2):67-72.

12. Seuntjens AD. Burnout in nursing--what it is and how to prevent it. Nurs Adm Q. 1982;7(1):12-19. doi:10.1097/00006216198200710-00005.
13. Navidian A, Masoudi G, Mousavi SS. Work-related Stress and the General Health of Nursing Staffs in Zahedans' Hospitals Emergency Wards (2004). J Kermanshah Univ Med Sci. 2005;9(3):17-26. [Persian].

14. Rahmani F, Behshid M, Zamanzadeh V, Rahmani F. Relationship between general health, occupational stress and burnout in critical care nurses of Tabriz teaching hospitals. Iran Journal of Nursing. 2010;23(66):54-63.

15. Dick MJ. Burnout in doctorally prepared nurse faculty. J Nurs Educ. 1992;31(8):341-346. doi:10.3928/0148-483419921001-04.

16. Oehler JM, Davidson MG, Starr LE, Lee DA. Burnout, job stress, anxiety, and perceived social support in neonatal nurses. Heart Lung. 1991;20(5 Pt 1):500-505.

17. Anisi J, Eskandari M, Ghorbani Z, Abdolmohammadi E. Personal and organizational factors related to job burnout in naval personnel. J Behav Sci. 2010;4(3):249-253.

18. Van Horn JE, Schaufeli WB, Greenglass ER, Burke RJ. A Canadian -Dutch comparison of teachers 'burnout. Psychol Rep. 1997;81(2):371-382. doi:10.2466/pr0.1997.81.2.371.

19. McCray LW, Cronholm PF, Bogner HR, Gallo JJ, Neill RA. Resident physician burnout: is there hope? Fam Med. 2008;40(9):626-632.

20. MohammadpoorasI A, Maleki A, Sahebihagh MH. Prevalence of professional burnout and its related factors among nurses in Tabriz in 2010. Iran J Nurs Midwifery Res. 2012;17(7):524529.

21. Filian E. Evaluation of burnout and its correlation with coping mechanisms in nurses of educational hospitals of Tehran [dissertation]. Tehran: Tehran Univ Med Sci; 1993.

22. Mahdizadeh $M$, Vafaei A, Taghipour A, Esmaeily $H$, Mahdizadeh SM, Ebrahimipour H. Occupational burnout and influence of work environment factors among family physicians in Khorasan Razavi. Occupational Medicine Quarterly Journal. 2014;5(4):42-51.

23. Abdulghafour YA, Bo-hamra AM, Al-Randi MS, Kamel MI, El-Shazly MK. Burnout syndrome among physicians working in primary health care centers in Kuwait. Alex J Med. 2011;47(4):351-357. doi:10.1016/j.ajme.2011.08.004.

24. Massoudi R, Etemadifar S, Afzali SM, Khayri F, Hassanpour Dehkordi A. The influential factors on burnout among nurses working in private hospitals in Tehran. Iran J Nurs Res. 2008;3(8-9):47-58. [Persian].

25. Mahmoodi GH, Rouhi GH, Moogerlou M, Rahmani Anaraki H. Relantionship of burnout in nursing with physical sitution and spicial in work environment. Ghorgan Med J. 2006;8(2):40-47. [Persian].

26. Koys DJ. The effects of employee satisfaction, organizational citizenship behavior, and turnover on organizational effectiveness: A unit-level, longitudinal study. Pers Psychol. 2001;54(1):101-114. doi:10.1111/j.1744-6570.2001. tb00087.x.

27. Talaei A, Mokhber N, Mohammad - Nejad M, Samari AA. Burnout and its related factors in staffs of university hospitals in Mashhad in 2006. koomesh J. 2008;9(3):237-246. [Persian].

28. Koivula M, Paunonen M, Laippala P. Burnout among nursing staff in two Finnish hospitals. J Nurs Manag. 2000;8(3):149158. doi:10.1046/j.1365-2834.2000.00167.x.

29. Frade Mera MJ, Vinagre Gaspar R, Zaragoza Garcia I, et al. [Burnout syndrome in different intensive care units]. Enferm Intensiva. 2009;20(4):131-140. doi:10.1016/s11302399(09)73221-3.

30. Santana Cabrera L, Hernandez Medina E, Eugenio Robaina P, Sanchez-Palacios M, Perez Sanchez R, Falcon Moreno R. [Burnout syndrome among nurses and nurses' aides in an intensive care unit and admission wards]. Enferm Clin. 2009;19(1):31-34. doi:10.1016/j.enfcli.2008.06.001. 